\title{
Effects of Inelastic Neutron Scattering in Magnetic Confinement Fusion Devices
}

\author{
Ivana Abramovic
}

\begin{abstract}
Components, surrounding the core of a magnetic confinement based fusion reactor, will be exposed to significant particle and heat fluxes that will cause severe (in many cases irreversible) damage of the components. In the D-T fusion reaction $80 \%$ of the energy is carried away by the $14 \mathrm{MeV}$ neutrons and the rest by the emitted alpha particles. Motion of neutrons is not restricted by the present magnetic field, which is why the damage they cause by interacting with surrounding materials is to a large extent inevitable. The greater the neutron flux to the material the larger the damage, and shorter the lifespan of the reactor component taking the flux. In order to make fusion economically viable it is important to increase the lifespan of the components since they are costly to produce, replace and dispose of. It is the premise of this work that neutron inelastic scattering plays an important role in neutron transport in MCF systems. This reaction mechanism has been overlooked in neutron transport calculations. Planned work entails modeling of inelastic scattering using reaction codes and results compared with experiment where possible. Data obtained will be further used in neutron transport calculations and in damage analysis of various materials in order to establish how significant inelastic scattering is for the viability of fusion energy production.
\end{abstract}

Keywords Inelastic neutron scattering - Nuclear reaction modeling - Magnetic confinement devices $\cdot$ Neutron transport • Irradiation damage

\section{Introduction}

Nuclear fusion offers a prospect of an inexhaustible source of energy and promises a reduction of environmental impacts of worlds increasing energy demand. The fact that scientific and engineering challenges, which have to be surmounted, haven't fully been foreseen in the beginning of fusion research has greatly contributed to

\footnotetext{
I. Abramovic ( $\square)$

Department of Nuclear Engineering, UC Berkeley, Berkeley, USA

e-mail: i.abramovic@berkeley.edu 
establishing the reputation of fusion power as an elusive goal. Some of the key issues that still have to be addressed are: understanding of turbulent transport in magnetized plasma's, suppression of edge localized modes, realization of an effective fuel cycle based on tritium breeding, improvement of particle and energy exhaust systems, development of the materials able to handle extreme conditions in the reactor. Materials can rightfully be singled out as the most critical issue. Large heat loads and particle fluxes cause melting, sputtering, erosion, re-deposition, swelling and displacements of atoms from their lattice structure. This further results in fuel dilution, changes in size of the components, changing of properties of the material.

In general effects of incoming neutrons result from three types of interactions with the target material:

absorption (transmutation)

elastic scattering

inelastic scattering

The first reaction is responsible for creating transmutation products (such as $\mathrm{He}$ ) that over time build up in the material. This consequently leads to deteriorating of material properties, making it swollen as the amount of helium increases, brittle as a result of accumulation of helium bubbles along grain boundaries, causing the change in thermal conductivity, activation of the material etc.

The second reaction is mostly responsible for damage expressed in terms of dpa-displacements per atom. Neutrons kick the lattice atoms out of their original position in the crystal lattice causing defects in the structure of the material by creating interstitials, substitutions and vacancies. ${ }^{1}$ Accumulation of such defects can even lead to phase transitions.

The third reaction is in the focus of my research. Knowledge of it is limited [1] yet it is important for modeling of $14 \mathrm{MeV}$ neutron interactions that originate from the D-T reaction. Change in the neutron energy spectrum along the propagation path will also be taken into account. Materials that are planned to be included in this analysis are the ones relevant for MCF: steel, tungsten, beryllium and carbon.

\section{Description of the Actual Work}

In order to address the problem of neutron transport and investigate the importance of inelastic neutron scattering both experimental and theoretical analysis will be conducted. Work can be divided into two conceptual parts. First part is related to nuclear reaction data evaluation while the second part deals with application of acquired knowledge to MCF systems.

\footnotetext{
${ }^{1}$ Types of defects in the lattice structure.
} 
Experiments will be conducted with the Bay Area Neutron Group at the 88 inch Cyclotron at Lawrence Berkeley National Laboratory. First set of data will be collected in gamma-gamma coincidence measurements of inelastic neutron scattering on iron 56. Scattering cross sections for this element are inadequately known for transport calculations and although the level schemes of iron are well documented the accuracy of the cross section measurements needs to be improved by $10-15 \%$ in the relevant energy range of $0.5-20 \mathrm{MeV}$ [1]. Reason for starting with iron 56, besides the fact that it is the major component of stainless and Euro-fer steels, is the fact that it has been singled out as a high priority isotope by the CIELO collaboration.

Measurements will take place in Cave 0 of the 88 inch Cyclotron facility. Four germanium detectors will be positioned roughly at an angle of $90^{\circ}$ with respect to the incident neutron beam. Coincident gammas will be measured in order to allow the use of data for level scheme building. Once the data has been collected and analyzed it will be compared with calculations. Different nuclear models allow properties such as the reaction cross sections to be calculated. These calculations are extremely complicated and cumbersome which is why computer codes have been developed to perform them. State of the art code EMPIRE allows selection of options and adjustment of input parameters such that the calculations best fit the experimental conditions and allows loading of measured data for direct comparison between evaluated data, measured data and theoretical predictions. Once the data from upcoming experiments is analyzed the result will further be used in the process of data evaluation to reduce the currently existing uncertainties in the energy range of interest.

The same reaction code will later on be used for modeling the inelastic scattering through various materials in MCF devices. Thickness of the target is not an input parameter of EMPIRE however satisfactory results can be obtained by multiple runs at different incident energies simulating the change in neutron energy spectrum. Calculations will be weighted by the build up factor for materials of interest and further supplemented by calculations taking into account the incident neutron flux. If inelastic scattering turns out to be a significant mechanism in fusion reactor environment full MCNP calculations will be necessary for precise modeling, these calculations are out of the scope of this project.

\section{Conclusions}

Research outlined in previous sections has not yet been performed. Therefore no definite conclusions or results can be presented at this point. However research question that will be answered once the project is complete is how significant is the mechanism of inelastic neutron scattering for neutron transport in fusion reactor environment. 
Acknowledgements Work planned has been made possible by the Bay Area Neutron Group in collaboration with UC Berkeley, Lawrence National Laboratory and University of Technology Eindhoven.

\section{References}

1. M.B. Chadwick et al., The CIELO collaboration: neutron reactions on $\mathrm{H}, \mathrm{O}, \mathrm{Fe}, \mathrm{U}$ and $\mathrm{Pu}$. Nucl. Data Sheets 118, I-25 (2014).

2. Reaction code obtained from, http://www.nndc.bnl.gov/empire/

3. M. Victoria, Structural materials for fusion reactors. EPFL-CRPP Fusion Technology Materials, CH-5232 Villigen PSI, Switzerland

4. G.R. Satchler, Introduction to Nuclear Reactions (Oxford University Press, New York, 1990)

Open Access This chapter is licensed under the terms of the Creative Commons Attribution 4.0 International License (http://creativecommons.org/licenses/by/4.0/), which permits use, sharing, adaptation, distribution and reproduction in any medium or format, as long as you give appropriate credit to the original author(s) and the source, provide a link to the Creative Commons license and indicate if changes were made.

The images or other third party material in this chapter are included in the chapter's Creative Commons license, unless indicated otherwise in a credit line to the material. If material is not included in the chapter's Creative Commons license and your intended use is not permitted by statutory regulation or exceeds the permitted use, you will need to obtain permission directly from the copyright holder. 